\title{
Improving the separation process of fine particles in drilling mud by ultrasonic waves
}

Afshin Akbari Dehkharghani*
Aggregation of solid particles in the drilling fluid has adverse effects on the drilling performance, including blocking drilling pipe, reducing fluid lubrication, and the blowout action. The purpose of this study was to prepare a solution for breaking the adhesion forces between the suspended solids and drilling fluid molecules. To investigate the effect of the ultrasonic waves on the separation of solid particles from reversed emulsion fluid, in vitro studies were conducted. Drilling mud was prepared in the form of different samples and the samples were then irradiated with ultrasonic waves for 2, 5, and $10 \mathrm{~min}$ and the intensities of 50,100 , and $150 \mathrm{~W} / \mathrm{m}^{2}$. To evaluate the stability of the emulsions and the efficiency of the separation process, caliper (volumetric) and density measurement methods were utilized. The results revealed the increased time and intensity of the ultrasonic radiation separates the phases and fine particles from the emulsion, and also increased the stability of reversed emulsion. The increased radiation time and intensity did not have any effect on the drilling mud and only delayed the optimal operation time and energy consumption.

\section{KEYWORDS}

Drilling fluid; reverse emulsion; ultrasonic.
*Corresponding Author:

Afshin Akbari Dehkharghani

Email:afshinkr@gmail.com

Tel.: +989121202754 radiations such as visible light cannot conduct this work [1]. Since ultrasonic wave sources are inexpensive, sensitive, and reliable, this provides a highly desirable way to probe and image the interior of opaque objects [2]. Ultrasound is divided into two main groups [1]:

1. High Frequency and Low Power Waves: The waves' frequency are at the range of 2-10 $\mathrm{MHz}$ and are used for performing analytical processes, such as specialized measurements (medical imaging and chemical analysis) [1].

2. Low Frequency and High-Power Waves: The waves are ranging from $20 \mathrm{kHz}$ to 100 $\mathrm{kHz}$ and are used in processes such as cleaning and welding of plastic materials due to their high power [1].

With regards to previous studies, the suitable frequency is in a range for the purification of solid particles is at the range of $20 \mathrm{kHz}$ to $40 \mathrm{kHz}$. Clearing process is divided 
into following two physical mechanisms: 1Cavitation; 2- Creating turbulent and rotational currents.

Interaction between the mechanical pressure and electrical force in an environment is called the piezoelectricity effect. Some crystals produce electrical force due to their mechanical pressure and, potential difference between two sides of same crystal, causing compression and expansion to continue as compression and expansion that generates the oscillation and wave [2]. The materials (crystals) with this specificity are called piezoelectric materials [3]. The piezoelectric effect is only found in crystals that do not have central symmetry. Quartz crystal is first material used to create the ultrasound waves [3]. Shape of the electric field at ionic dimensions is demonstrated in Figure 1. Figure 1 shows how the electric field is formed by applying the mechanical force for the crystal [4].
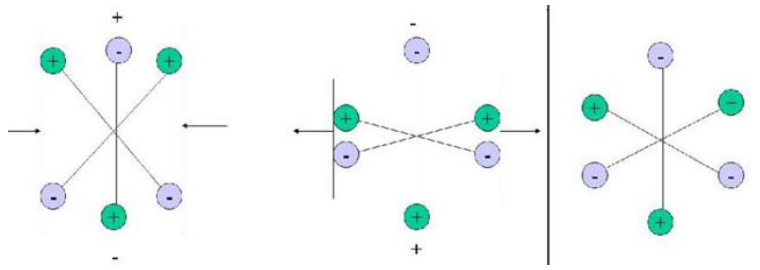

FIGURE 1 A unit of quartz crystal structure and the effect of stress on its electric field production

This technology uses piezoelectric converters for converting electrical energy into ultrasound. The desired frequency was provided based on the physical properties such as the angle of cut, thickness, crystal material and the applied voltage [2]. The technology is usually ceramic, which is coated with lead zirconate and a silver coating on the interior and exterior.

Using ceramic piezoelectric materials has advantages, such as to be synthetic that can be produced for the desired size and properties that are much cheaper and highly efficient in converting the electrical energy into acoustic [4].
Jalilian et al. [5] investigated ultrasonicassisted micro solid phase extraction of arsenic on a new ion-imprinted polymer synthesized from chitosan-stabilized pickering emulsion in water, rice and vegetable and could investigate arsenic analysis was performed using pickering emulsion method in well water, rice, and also vegetable samples. Vinodhkumar et al. [6] investigated reduced graphene oxide based on simultaneous detection of neurotransmitters and showed that the proposed sensor could be applied for the real samples. El-Khateeb [7] investigated the different horizontal subsurface substrates in flow wetlands and reported that properties of the effluent improved significantly as the effluents passed by the wetland cell and the quality of the effluent along the flow treatment path was improved.

\section{Construction of emulsion drilling fluid}

Reverse emulsion or petroleum fluid is prepared by mixing the oil-to-brine volume ratio of 9: 1 (excluding emulsifier volume) and emulsifier with concentration of $8 \mathrm{~L} / \mathrm{m}^{3}$. In this emulsion, $10 \%$ of the brine is made up of $30 \%$ by weight of $\mathrm{CaCl}_{2}$ salt. Shortly thereafter, $10 \mathrm{~kg} / \mathrm{m}^{3}$ of the lime and $25 \mathrm{~kg} / \mathrm{m}^{3}$ of Organophilic clay are added. Selected microparticles are then added into fluid in an amount of $0.1 \mathrm{~g} / \mathrm{mL}$. The fluid is mixed with a commercial mixer per time and for $5 \mathrm{~min}$ and the derivatives are then added. The steps for preparing the reverse emulsion are summarized as follows [8].

1. The base oil and the primary emulsifier are mixed with concentration of $8 \mathrm{~L} / \mathrm{m}^{3}$ for $5 \mathrm{~min}$ at the speed of $10000 \mathrm{rpm}$ and room temperature [8].

2. The lime is added to dish and blended for 5 min at a speed of $10000 \mathrm{rpm}$.

3. Brine is added and blended at speed 10000 rpm for 5 min.

4. Organophilic clay is added and blended for $5 \mathrm{~min}$ at a speed of $17000 \mathrm{rpm}$. 
5. Emulsion is stored at room temperature for $24 \mathrm{~h}$.

6. Solid microparticles are added into the amount of $0.1 \mathrm{~g} / \mathrm{mL}$.

7. The emulsions prepared for each type of fuzzy separation are controlled and visualized.

8. Emulsions should be mixed again at speed of $10000 \mathrm{rpm}$ before each test.

9. For three different times, and three different ultrasound intensities, the experiment is repeated on the prepared samples to determine the effect of this technology on the separation of fine particles. At each stage of emulsion stability, density and viscosity are measured as reported below [2].

Reversed emulsions, which are considered as samples for drilling fluid, contain fine particles that are dispersed in the fluid. Suspended particles in these drilling fluids generally do not settle. In other words, a stable emulsion is an emulsion that does not detach phases after formation over time [4].

The primary purpose of this experiment is to find out whether the emulsions prepared after the technology removal process still need to be separated. In other words, the primary purpose is to determine the separation rate for different intensities of ultrasound [9].

\section{Effective parameters in emulsion fluid stability}

Parameters affecting on emulsion stability, including emulsion $\mathrm{PH}$, drilling fluid temperature, electrolyte composition effect, drilling fluid rolling process, Organophilic clay constituent composition, and wettability. For these parameters a steady state is considered. To investigate the effect of time and intensity of ultrasound radiation on the removal of fine particles from drilling, fluid under reverse emulsion conditions is investigated [10].

\section{Caliper measurement (Volumetric)}

Digital calipers are used for assessing phase separation. For specific samples, the separated layers are indistinguishable and face measurement process with difficult that cause uncertainties in the measurements. Height of the sediment and oil (oil) floating on the surface is measured by a digital caliper in $\mathrm{mm}$ and measured with the aid of the following formulas are converted to $\mathrm{mL}$ [11]:

If $h<17.23$

$$
V=\left[\frac{0.362 \pi h \times(h+4.04)^{2}-\pi h r_{1}^{2}}{3}\right]
$$

Otherwise:

$$
V=\left[\pi(h-17.23) \times r_{0}^{2}\right] \times 10^{-3}+V_{0}
$$

where $r_{0}(\mathrm{~mm})$ is the pipe diameter, $h$ $(\mathrm{mm})$ is the separated layer altitude, $\mathrm{r}_{1}(\mathrm{~mm})$ is the incomplete cone upper diameter, and $\mathrm{V}_{0}(\mathrm{~mL})$ is the incomplete cone volume (Figure 2).

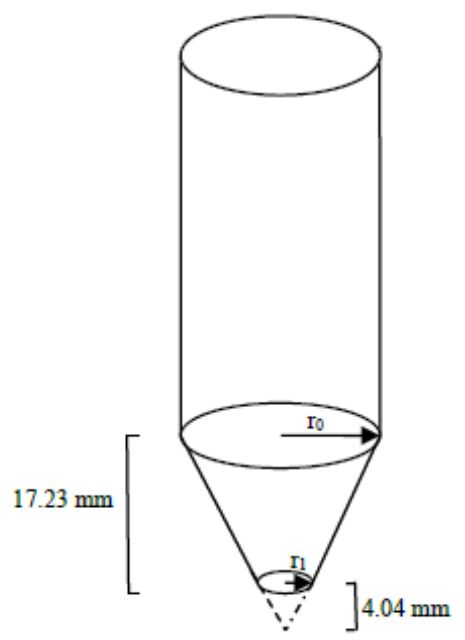

FIGURE 2 Schematic representation of the parameters and values used in volumetric calculations

The values obtained from the caliper measurements are converted to volume as the centrifuges pipe is not cylindrical. In other words, the phase separation height ratio is not a good indicator for estimating the phase separation as the sediment fraction 
ratio is calculated more than the actual amount. Height ratio scale is used as an indicator, the volume of the fraction the cone of the centrifuge tube will be less than the cylinder of equal height. So, the altitude index is not the right index.

An example of phase separation experiments to determine single regions in this reverse emulsion is demonstrated in Figure 3. Generally, a transparent layer of oil is seen at the top of the sample. The transition zone 1 is the reverse emulsion beneath the oil floating layer, and the transition zone 2 is the unbound emulsion containing organic clay soil [12].

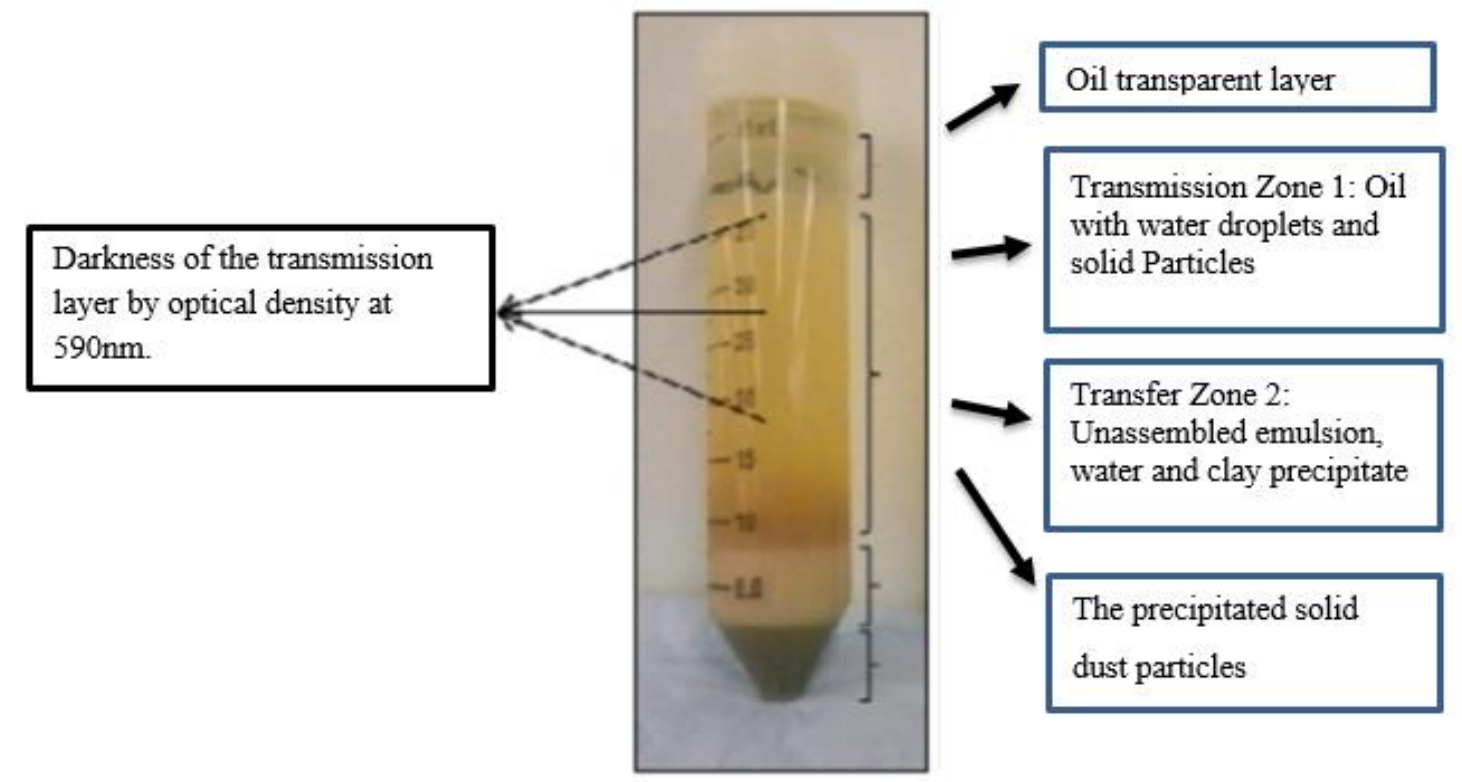

FIGURE 3 Two-phase separation for post-centrifugal neodryl emulsion sample

Deposition is mostly due to its dark color and subsidence at the bottom of the pipe, mostly containing dust and solid particles. Each layer may contain both phases, although the fraction of this value is not known. Some measurements are within the given range because the surface of the layers separated is not smooth enough. Caliper measurements have means over the observation range. The converted volumes of the preceding equations are calculated in the following formulas for calculating the volume ratios and fractions [13].

$R_{\text {oil }}=\frac{\text { Total volume of oil separated }(\mathrm{mL})}{\text { total volume }- \text { sediment volume }(\mathrm{mL})}$

(3)

$$
\left.R_{\text {sediment }}=\text { (sediment volume }(\mathrm{mL})\right) /
$$

(Volume of solid particles and dry dust $(\mathrm{mL}) *$

At the denominator of the oil ratio (Equation 3 ), the volume of sediment decreases from the total volume. For example, if a certain volume of dust and fine particles is added into $300 \mathrm{~mL}$ neodryl emulsions, the volume of sediment isolated for higher concentrations of fine-grained particles is higher than for lower concentrations of these particles. The volume fraction of the solid particles was also obtained by dividing the volume of the precipitate by the volume of the primary solid powders. This ratio represents the volume of fine particles deposited on the sediment particles added at beginning of work [14].

\section{Fluid electrical stability}

Electrical Stability Reverse emulsion is performed by OFITE electrical stability meter. The probe is immersed in the sample and shaken for $10 \mathrm{~s}$ to form a compound at uniform temperature. The voltage in emulsion breaks is read [15]. 


\section{Ultrasonic wave radiation}

In this experiment, the probe was used with varying the radiation intensity up to a maximum nominal intensity of $1000 \mathrm{~W} / \mathrm{cm}^{2}$. The samples were washed in the bath, respectively, and sonicated at 50, 100 and $150 \mathrm{~W} / \mathrm{m}^{2}$ intensities for 2,5 , and $10 \mathrm{~min}$. Nine experiments were performed for separating solid microparticles and 9 experiments were performed for determining the effect of ultrasound on this separation. Phase separation is immediately visualized, and the volume of sediment and floating oil can be determined indirectly by digital calipers $[16,17]$.

\section{Statistical analysis}

The mean were calculated and indicated in figures by vertical error bars to describe the distribution of the measurements. To study the significance of the results, two-way

\section{Obtained results}

Following radiation ultrasonic waves, cavitation bubbles enclosing suspended particles, thereby separating them from the drilling fluid and bringing them to the sample surface. Accordingly, micron-scale fine particles are separated by bubbles created from the reverse emulsion. Gradually, with the passage of ultrasonic radiation, the rate of separation of fine solids is increased. After irradiation, it is observed that the oil was deposited at the bottom of the sample due to its lower density and the fine particles are deposited. Between these two phases, a drilling fluid, part of which is separated from the oil and fine particles, is known as the transition zone. This way, the amount of fine particulate matter isolated can be calculated by caliper or volume measurements. This phase separation rate in terms of volume fraction and for different irradiation times is

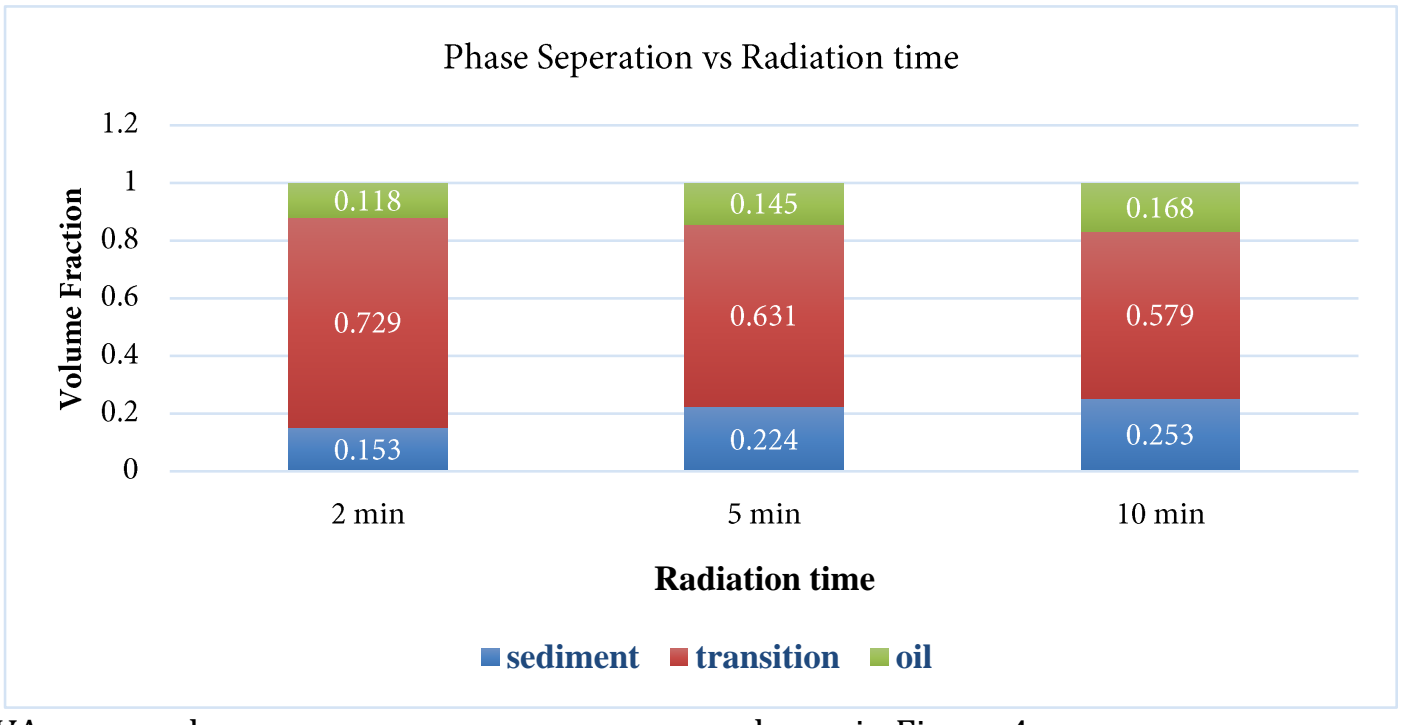

ANOVA was used.

shown in Figure 4.

FIGURE 4 Separation diagram of drilling fluid phases in terms of ultrasonic irradiation time with radiation intensity of $100 \mathrm{w} / \mathrm{m}^{2}$

As seen in Figure 4, the increased ultrasonic radiation time enhanced the amount of the solid sediment particles separated from the drilling fluid; however, the volume fraction of the oil phase increased. It indicated that, increasing the irradiation time enhanced the separation of more finegrained particles. 
The deposition rate was $0.071 \mathrm{v} / \mathrm{v}$ after the time increased from $2 \mathrm{~min}$ to $5 \mathrm{~min}(3$ min interval), but due to increased time from $5 \mathrm{~min}$ to $10 \mathrm{~min}$ ( $5 \mathrm{~min}$ interval), the amount of particulate matter is to be $0.029 \mathrm{v} / \mathrm{v}$. Increasing the irradiation time can enhance the amount of fine particle separation but always depending on the sample size tested, the amount of fine particles present in the sample. The type of drilling fluid has an optimal radiation time. This optimum irradiation time has two characteristics. Firstly, it separates the desired amount of fine particles. Secondly, it reduces energy consumption and process runtime as much as possible. Following the passage of time (from
5 min onwards), the effect of ultrasonic radiation time on the removal of solid particles decreases, that is, with the passage of ultrasonic radiation time. The rate of irradiation time on solid particle separation is decreasing. In the current example, radiation practically no more than $10 \mathrm{~min}$ of financial justification is economical since virtually no ultrasound radiation will have a significant impact on remediation.

The higher force of ultrasound radiation and the strength of the ultrasound waves on the drilling fluid (reversed emulsion), the greater the fine particle removal. This is illustrated in Figure 5.

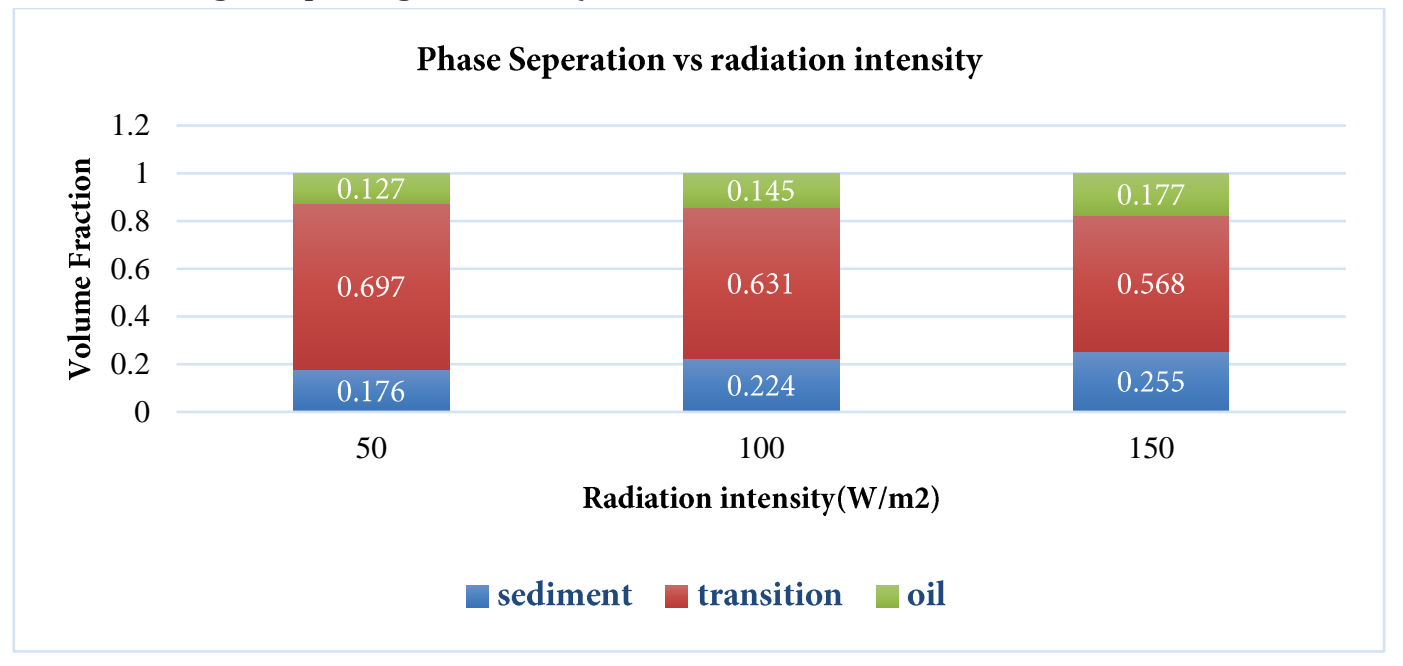

FIGURE 5 Phase separation in terms of different radiation intensities (5 min radiation time)

As the intensity of the ultrasound radiation increases, the particle solid and oil phase separation increase that indicate the significant effect of radiation intensity on the rate of sediment separation. Since the emission of waves with higher intensity requires more energy consumption, the results for the optimal radiation intensity should be considered for the reverse emulsion (Figure 6). The effect of the radiation intensity on the separation rate is also gradually decreasing, which is due to decreased concentration of the fine particles in the drilling fluid over time. Increasing the radiation intensity from 50 to $100 \mathrm{w} / \mathrm{m}^{2}$ enhanced the volume of the separated sediment volume by 0.048 , while increasing this amount from 100 to $150 \mathrm{w} / \mathrm{m}^{2}$ volume of sediment separated by 0.031 . It means the amount of sediment in the sample was decreased through the effect of ultrasound on the removal of fine particles. For instance, in the case of the reversed emulsion sample, a concentration of $50 \mathrm{~g} / \mathrm{L}$ increased the intensity of radiation by no more than 150 $\mathrm{w} / \mathrm{m}^{2}$ rational and only increased the costs. It may even cause the loss of new chemical bonds, and virtually abrupt changes in the properties of the drilling fluid and thus have devastating effects. In such a way that due to changes in drilling fluid properties and reducing the desired viscosity, prevent the 
drilling fluid from drilling properly and reduce the efficiency of operation or prevent the drilling fluid from maintaining proper stability of the drilling wall.

Therefore, it was found that ultrasonic irradiation (time and intensity of radiation) as two important parameters are very efficient in the separation of fine particles. Ultrasonic technology is very successful for removing these drill chips that have detrimental effects on drilling operations and well stability. The presence of these drill chips causes the drilling pipe to disrupt the drilling operation, and may increase the viscosity and damage to the wells by increasing the local viscosity in the emulsion. So, ultrasound acts as an efficient technology for removing these chips.

The investigation of the effect of ultrasonic waves on electrical stability

The results of the electrical stability test for the samples are given in the following figures. Virtually abrupt changes in the properties of the drilling fluid and thus have devastating effects. In such a way that due to changes in drilling fluid properties and reducing the desired viscosity, prevent the drilling fluid from drilling properly and reduce the efficiency of operation or prevent the drilling fluid from maintaining proper stability of the drilling wall.

Therefore, it was found that ultrasonic irradiation (time and intensity of radiation) as two important parameters are very efficient in the separation of fine particles. Ultrasonic technology is very successful for removing these drill chips that have detrimental effects on drilling operations and well stability (Figure 7). The presence of these drill chips causes the drilling pipe to disrupt the drilling operation, and may increase the viscosity and damage to the wells by increasing the local viscosity in the emulsion. So, ultrasound acts as an efficient technology for removing these chips.
The investigation of the effect of ultrasonic waves on electrical stability

The results of the electrical stability test for the samples are given in the following figures

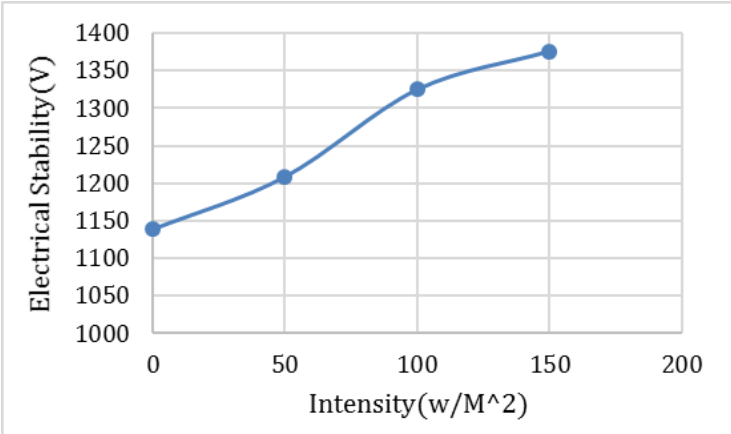

FIGURE 6 The effect of ultrasound intensity on electrical stability of drilling mud

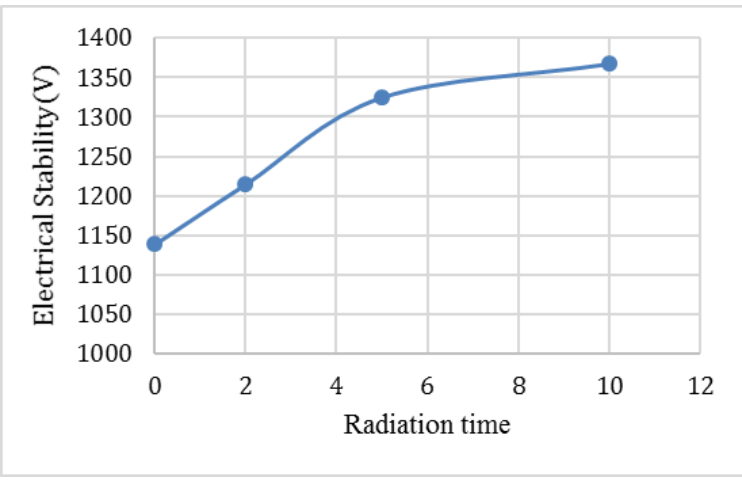

FIGURE 7 The effect of ultrasonic radiation time on mud electrical stability

Increasing the time and intensity of ultrasound enhanced the read voltage, indicating that the radiation of the ultrasound to the sample increased the stability of the drilling mud samples.

\section{Conclusion}

The results showed that ultrasonic technology is suitable for the removal of fine particles in drilling fluid. It was found that, that increasing the irradiation time and the intensity of irradiation generally resulted in more phase separation in the reversed emulsion samples and to be a greater extent separates the fine particles and oils. In 
addition, ultrasonic radiation improved the stability of the prepared emulsion.

In addition, it should be noted that the time and intensity of the radiation are optimal as they gradually lose their effect on the drilling mud and practically will only delay the time of operation and energy consumption from the optimum level. The results of this study were as follows:

1. As the ultrasonic radiation time increases, the rate of separation of the sediment and oil phases from the drilling fluid increases.

2. As the intensity of the ultrasound radiation increases, the separation of the sediment and oil phases increases

3. Increased the time and intensity of the ultrasonic radiation increases the measured voltage stability. It means that increased the intensity and time of ultrasound increases the stability of the samples.

4. Enhancing the timing and intensity of the ultrasound radiation shifted the average particle size to smaller numbers. However, over time the optimum radiation intensity (for example in this sample tested after 10 minutes and intensity of $150 \mathrm{w} / \mathrm{m}^{2}$ ) decreases the sonication effect on the solid particle separation rate.

5. Ultrasonic waves have promising results in phase separation. It was found that these waves of water dry up the colloidal particles and thus allow them to contact and sit side by side.

6. Ultrasonic proved to be very effective in stacking particles. The accumulated particles were visible to the naked eye after the sonication process. Several mechanisms are responsible for describing particle collisions: orthokinetic, acoustic wake effect, and microbubble cross-pressure contact mechanisms also play important roles in causing considerable turbulence and turbulence in the fluid, which increases the likelihood of particle collisions and accumulation. The addition of clay particles further reduces degradation because they reduce the velocity of inter-particle collisions, and thus reduce the accumulation of particles.

7. To scale this technology, a careful examination and linkage in ultrasonic engineering is required to fully optimize the operation frequency and the output power.

8. In sum, the results showed that, increased time and intensity of ultrasonic radiation separates the phases and fine particles from the emulsion, and increases the stability of reversed emulsion.

\section{Acknowledgements}

The authors would like to thanks the central Tehran Branch of Islamic Azad University, Tehran, Iran.

\section{Orcid:}

Amin Amraei: https://orcid.org/0000-00020012-4180

Afshin Akbari Dehkharghani:

https://orcid.org/0000-0002-9241-1354

\section{References}

[1] T. Wiersberg, R. Kietäväinen, L. Ahonen, I. Kukkonen, S. Niedermann, EGU Gen. Assemb. Conf. Abst., 2014, 16, 312-323.

[2] D.N. Cheeke, CRC Press LLC. 2002, pp.17.

[2] H. Xu, X. Jian, T.T. Meek, Q. Han, Essent. Read. Light. Metal., 2013, 3, 246-250.

[3] J.L. Rose, Cambridge University Press, 2004, 121-128.

[4] J.B. Pawley, Springer, 2006, ISBN 0-38725921-X.

[5] R. Jalilian, M. Shahmari, A. Taheri, K. Gholami, Ultrason. Sonochem., 2019, 61, 1-27.

[6] G. Vinodhkumara, R. Ramyab, M. Vimalanc, I. Vetha Potheherd, A. Cyrac Peter. Prog. Chem. Biochem. Res., 2018, 1, 40-50.

[7] A.Y. El-Khateeba, M.H. Mahmoudb, M. Fakih. Prog. Chem. Biochem. Res. 2019, 2, 2023.

[8] B.G. Park, I.J. Park, J.S. Han, S.M. Lee, C.G. Lee, C.S. Ha, J. Disper. Sci. Technol., 2013, 34, 560-565. 
[9] E. Riera-Franco de Sarabia, J.A. GallegoJuarez, G. Rodriguez-Corral, L. Elvira-Segura, G. Gomez, Ultrason., 2000, 38, 642-646. [10] R.J. Leu, M.M. Ghosh, J. Am. Water. Work. Assoc., 1988, 8, 159-167.

[11] J.K. Vasshus, M. Trond, U.S. Patent No. 8, 746, 460, 2014.

[12] A.R. 13B-1, American Petroleum Institute, 2005.

[13] W. Chantrapornchai, F. Clydesdale, D.J. McClements, Colloid. Surface. Physicochem. Eng. Aspect, 1999, 155, 373-382.

[14] H. Hamidi, E. Mohammadian, M. Asadullah, A. Azdarpour, R. Rafati, Ultrason. Sonochem., 2015, 26, 428-436.

[15] S. Levine, E. Sanford, Can. J. Chem. Eng., 1985, 63, 258-268.
[16] R. Jiang, X. Li, L. Zhang, X. Xu, Inform. Technol. J., 2013, 12, 6817-6821.

[17] B.C. Crittendon, U.S. Patent No. 2, 859, 404, 1958. Washington, DC: U.S. Patent and Trademark Office.

How to cite this article: Amin Amraei, Afshin Akbari Dehkharghani*. Improving the separation process of fine particles in drilling mud by ultrasonic waves. Eurasian Chemical Communications, 2020, 2(6), 722730.

Link: http://www.echemcom.com/article_10543 4.html

Copyright () 2020 by SPC (Sami Publishing Company+ is an open access article distributed under the Creative Commons Attribution License, which permits unrestricted use, distribution, and reproduction in any medium, provided the original work is properly cited. 